\title{
Pulsed-field magnetometry for rock magnetism
}

\author{
Kazuto Kodama
}

\begin{abstract}
An improved method is proposed for measuring dynamic magnetizations of bulk volcanic rock samples induced by a pulsed-field of $0.7 \mathrm{~T}$ and a duration of $10 \mathrm{~ms}$. The transient magnetization is measured by a sensing system that consists of a pair of inductive differential coils, an analog preamplifier and integrator, and a high-speed digital storage scope. The system was calibrated using a paramagnetic salt $\left(\mathrm{Gd}_{2} \mathrm{O}_{3}\right)$ and was tested to different kinds of volcanic rocks with their magnetic properties well-documented previously. The results were comparable with those measured by a quasi-static method using a vibrating sample magnetometer, although there were small discrepancies in hysteresis parameters suggesting the time-dependence of the magnetic properties. The proposed system provides not only the magnetization over the short interval of a pulse but also the rapid ( $\sim 3 \mathrm{~ms})$ exponential decay after a pulse. The decay time constant was different among the samples under study, indicating the variations of their magnetic relaxation time. Although the present system is not sensitive enough to characterize varieties of natural samples including sediments, it has the potential as a versatile and convenient tool for rock magnetism.
\end{abstract}

Keywords: Rock magnetism; Pulsed-field; Magnetometry; Dynamic magnetization; Hysteresis

\section{Correspondence/findings Introduction}

Measurement of magnetizations during a short interval (1-10 ms) of a pulsed field generated by pulse magnet has long been employed in various fields of experimental physics (e.g., Grössinger 1982; Grössinger et al. 1993; Miura 2007; Lv et al. 2013). Since the invention of the pulse magnet by Kapitza (1924), magnetometry using the pulsed field up to $100 \mathrm{~T}$, the pulsed field magnetometry (PFM), has led to a variety of applications, such as NMR, magnetostriction, magneto-optical spectroscopy, and characterization of permanent magnets (e.g., Ludwig et al. 2002; Dudding et al. 2002; Haase et al. 2004; Meier et al. 2011). Unlike the static-field techniques using superconducting magnets, PFM is cost-effective and reliable for measuring the magnetization of samples in high fields in the 10-T range (e.g., Grössinger 1982). Pick-up coil systems are generally used in PFM, and the derivative signal of the magnetization is useful especially for the identification of the phase change of magnetic

Correspondence: kdma@kochi-u.ac.jp

Center for Advanced Marine Core Research, Kochi University, Nankoku, Kochi 783-8502, Japan materials in high fields (e.g., Mizutani et al. 1998; Nojiri et al. 2007; Takeyama et al. 2012).

The pulse magnet by itself has also been widespread in rock magnetic studies as a convenient apparatus for the rapid acquisition of isothermal remanent magnetization (IRM). The IRM unmixing analysis has become a conventional tool for the quantitative characterization of magnetic minerals (Kruiver et al. 2001; Heslop et al. 2002; Egli 2004; Abrajevitch and Kodama 2011). However, the measurement of dynamic magnetizations in pulsed fields has been paid little attention except the pioneering work by Radhakrishnamurthy and Likhite (1987) who obtained hysteresis parameters from a set of synthetic and basalt samples using a laboratory-made PFM system (Likhite et al. 1985). They compared the results both at room and low temperature (77 K), suggesting that the latter provided a better estimate of the composition and domain state. Recently, Kodama (2015) developed a simple instrumentation consisting of a pickup coil, a digital storage scope, and a commercially available pulse magnet. This new system was applied to a set of synthetic and volcanic rocks. The results indicated the dynamic magnetization behaviors in a short duration of pulse $(10 \mathrm{~ms})$ as well as the rapid $(3 \mathrm{~ms})$ relaxation 
behaviors after pulses that have not been obtained by conventional static-field methods. However, due to the sensitivity constraint, samples to be measured needed to be strongly magnetic, in terms of the induced magnetization moment, above the $10-\mathrm{mAm}^{2}$ range.

This study is an attempt to improve the previous system (Kodama 2015) by increasing the sensitivity sufficiently to measure a wide spectrum of igneous rocks containing titanomagnetite or other magnetic minerals with high saturation magnetization $\left(M_{\mathrm{s}}\right)$. Based on the results from the earlier works (Radhakrishnamurthy and Likhite 1987; Kodama 2015), an improved system with approximately 1 order of magnitude better sensitivity was developed. This system was tested to a set of igneous rocks with the magnetic properties well-documented by previous works. These results, along with the advantage and disadvantage of the new instrumentation, were provided. Finally, the still remaining limitations and constraints were described, and ideas and methods in order to make PFM as a convenient tool for rock magnetism were proposed.

\section{Methods}

In order to measure magnetizations in pulsed-fields, an inductive method using a pick-up coil located in a pulse magnet is generally employed. To measure the rapid magnetic flux change of a sample in a strong pulsedfield, it is necessary to construct a pick-up coil system that is compensated, usually in the accuracy of $<10^{-5}$. A number of techniques have been proposed to achieve this requirement (e.g., Grössinger 1982; Grössinger et al. 1993; Dudding et al. 2006). We employed in this study the configuration by Kodama (2015) using a coaxial pick-up coil pair located in the sample cavity of a commercial pulse magnet (ASC IM10-30 impulse magnetizer).

In the previous system, the coil output was acquired directly by a fast digital oscilloscope and then transformed to a computer for numerical integration. The results suggested that it could be a promising tool for rock magnetism, but the sensitivity was still insufficient to be used as an alternative to the conventional, quasi-static method. This required the development of an improved version with better sensitivity by adding an analog circuit for the amplification and integration. The preamplifier has four selectable gains $(\times 2, \times 5, \times 10$, and $\times 20)$ and a following integration circuit (Fig. 1). The inclusion of this circuit increased the system sensitivity by an order of magnitude than the previous one by means of selecting an appropriate gain according to a sample magnetization. The new system is more flexible and cost-effective than the previous system in that, if desired, the integrated signal can be acquired with an analog-to-digital converter installed in a computer, without an expensive fast digital oscilloscope for data acquisition and numerical integration.
The key to constructing the pick-up coil system is to achieve the accurate compensation of the two oppositely wound coils. It is expected that a larger number of coil windings would result in higher sensitivity. However, as discussed by Kodama (2015), the stray capacitance and the impedance of the pick-up coils increase with an increasing number of coil windings. This leads to a highfrequency resonance of the pick-up coil for the short interval $(<1 \mathrm{~ms})$ of the rise and fall of a pulse. The resonance is stronger with higher pulsed fields, resulting in the lower signal-to-noise ratio (SNR). This problem was already pointed out by Likhite et al. (1985) who was forced to limit the pulse peak $<0.6 \mathrm{~T}$ due to the imbalance of pick-up coil caused by "ringing" of the coil system. In the same way, because a slight failure of the coil compensation contributes to greater reduction of the SNR, we found that the maximum peak of a pulse was set to $0.7 \mathrm{~T}$ in this study. In addition, the measurement without a sample was performed for each run, and this background signal was subtracted from the signal with the sample.

\section{Samples}

The present system achieved the better sensitivity in this way, requiring no averaging by a number of repeated measurements of one sample as was necessary for the previous system. This advantage allowed more accurate calibration using paramagnetic oxide salts, such as $\mathrm{Gd}_{2} \mathrm{O}_{3}$, a standard sample for the calibration of conventional susceptometers. Ferromagnetic substances such as nickel are often used for calibration, but we consider this kind of paramagnetic powder more appropriate because the paramagnetic magnetization that is proportional to field is idealistic to test the rapid response of the PFM system. We used commercially available $\mathrm{Gd}_{2} \mathrm{O}_{3}$ powder (8.99 g, 99.9 \% purity, Rare Metallic Inc., Tokyo) glued with epoxy in a 7-cc plastic cube. Using this $\mathrm{Gd}_{2} \mathrm{O}_{3}$ sample, we made an inter-calibration between our system and a magnetic property measurement system (MPMS) that was independently calibrated using a palladium crystal, a paramagnetic rare-earth metal. We measured a set of natural samples including four different kinds of Quaternary volcanic rocks (OS2-2, MY07-2, DS04-2, SW03-07) and one Paleozoic granite (SH03-07). These samples were selected on the grounds that their hysteresis measurements were well-documented by previous studies (Yamamoto and Hoshi 2008; Yamamoto et al. 2010; Kodama 2013; Kodama 2015). They indicate that three of them (DS04-2, SW03-07, SH03-07) were saturated below $0.7 \mathrm{~T}$ (the pulse peak in this study), while the other two (OS2-2, MY07-2) showed no saturation plateau due to the superimposition of paramagnetic components. In terms of the domain structure, the granite $\mathrm{SH} 03-07$ is dominated by MD particles and the 


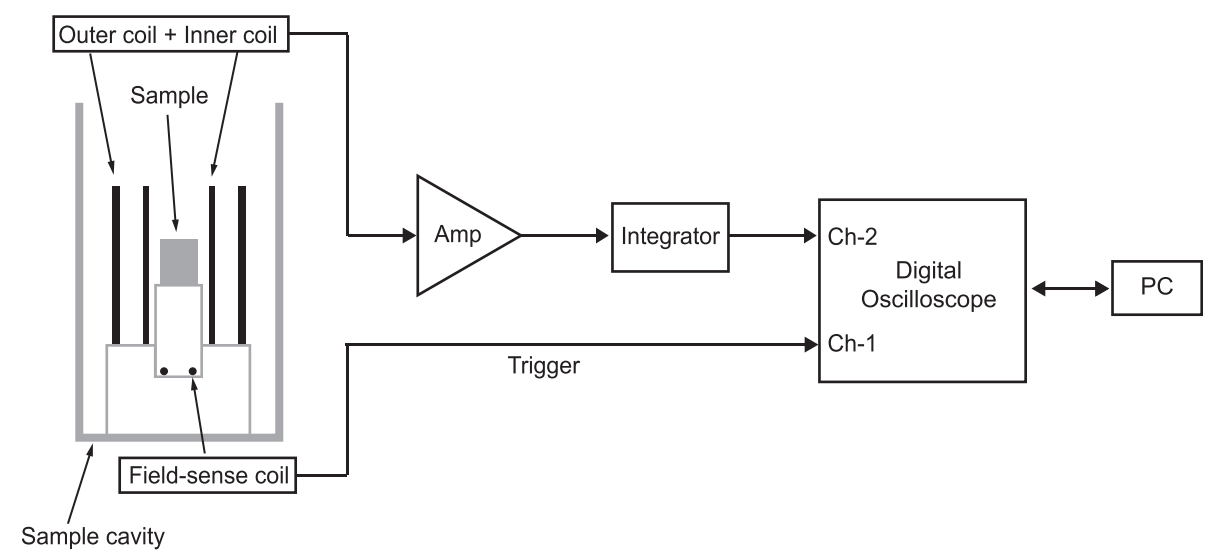

Fig. 1 Schematic diagram of the measurement system. A pulse from the field-sense coil triggers to acquire the signal from the coaxial pick-up coils through the analog circuit comprising a preamplifier and integrator

remaining samples are in the PSD range. We measured these samples to see if the PFM system in this study could discriminate the various magnetic properties and how the PFM results would be different from those of the static-field measurements.

\section{Results and Interpretations}

Figure 2 shows the results from $\mathrm{Gd}_{2} \mathrm{O}_{3}$ obtained by the present system and a MPMS. The magnetization vs. time $(M-t)$ curve and the field vs. time $(H-t)$ curve, being scaled to their maximum peaks, have an equivalent shape (Fig. 2a). This led to the magnetization vs. field $(M-H)$ plot in Fig. 2b, which is linear in the same manner as that obtained by the MPMS (inset). This allowed calibration by using the two $M-H$ lines with different $y$-axis units being volt $/ \mathrm{kg}$ in the present system and $\mathrm{Am}^{2} / \mathrm{kg}$ by the MPMS. This yielded the calibration constant of $25.5 \mu \mathrm{Am}^{2} / \mathrm{mV}$. Using this value, the raw data in this study were converted into magnetic moment per unit weight $\left(\mathrm{Am}^{2} / \mathrm{kg}\right)$ in the following figures.

Figure 3 is an example demonstrating the better sensitivity of the present system using sample OS2-2. In this figure, the mean $M-t$ curve and associated errors obtained by averaging over 10 times are shown for the present (Fig. 3a) and previous (Fig. 3b) systems. It is obvious that the mean $M-t$ curve of the former is smoother and the associated error range is more reduced than of the latter. In addition, the improvement in the same way was achieved for the portion of the $M-t$ curve after switching off the pulse.

Each of the $M-t$ curves of the five samples that were observed by a single measurement run are plotted in Fig. 4. The flattening of the curves from the three samples (DS04-2, SW01-17, SH03-07) show their saturation for the interval from about $1 \mathrm{~ms}$ after starting of a pulse. In contrast, the other two (OS2-2, MY07-2) have the $M-t$ curves with no such flattening but gradual peaks at around $4 \mathrm{~ms}$ when the field reached the maximum. It is noted that all of them showed more or less a tail-off starting immediately after switching off a pulse. Similar decay patterns have already been reported for selected synthetic materials and the volcanic rocks in the PSD range and interpreted as magnetic relaxation (Kodama, 2015). It is also noted in Fig. 4 that sample SH03-07 in the MD range showed a similar relaxation behavior. Thus, it is suggested that this kind of relaxation may be a ubiquitous phenomena for the igneous rocks ranging in the PSD to MD range. This issue is discussed in more detail in the following section.

Figure 5 shows the individual $M-H$ curves obtained from the combination of the $H-t$ curve and the $M-t$ curves in Fig. 4. Each of them consists of two curves that could correspond to an initial magnetization curve and a branch of one hysteresis loop in a decreasing field measured with a vibrating sample magnetometer (VSM). Although the rate of field change is about 3 orders of magnitude faster in a pulse than in a VSM, the patterns of the $M-t$ curves in Fig. 4 can be observed in the corresponding $M-H$ curves in the same way as in typical hysteresis loops. The magnetic saturations initiated around $0.3 \mathrm{~T}$ for samples DS04-2 and SW01-17, while they are not observed for the others. Comparing these results with the corresponding VSM hysteresis curves by previous studies (Yamamoto and Hoshi 2008; Yamamoto et al. 2010; Kodama 2013; Kodama 2015), no significant difference was observed in their patterns. However, it appears that the irreversibility of the $M-H$ curves in Fig. 5 is slightly wider than the hysteresis curves measured on a VSM. This seems to be more obvious for SH03-07 because its VSM hysteresis curve is saturated and reversible with the considerably small $M_{\mathrm{rs}} / M_{\mathrm{s}}=0.02$ (Kodama 2013). In contrast, the $M-H$ curve of SH03-07 is slightly irreversible and the ratio that corresponds to the conventional $M_{\mathrm{rs}} / M_{\mathrm{s}}$ is 0.06 . We interpret that these 

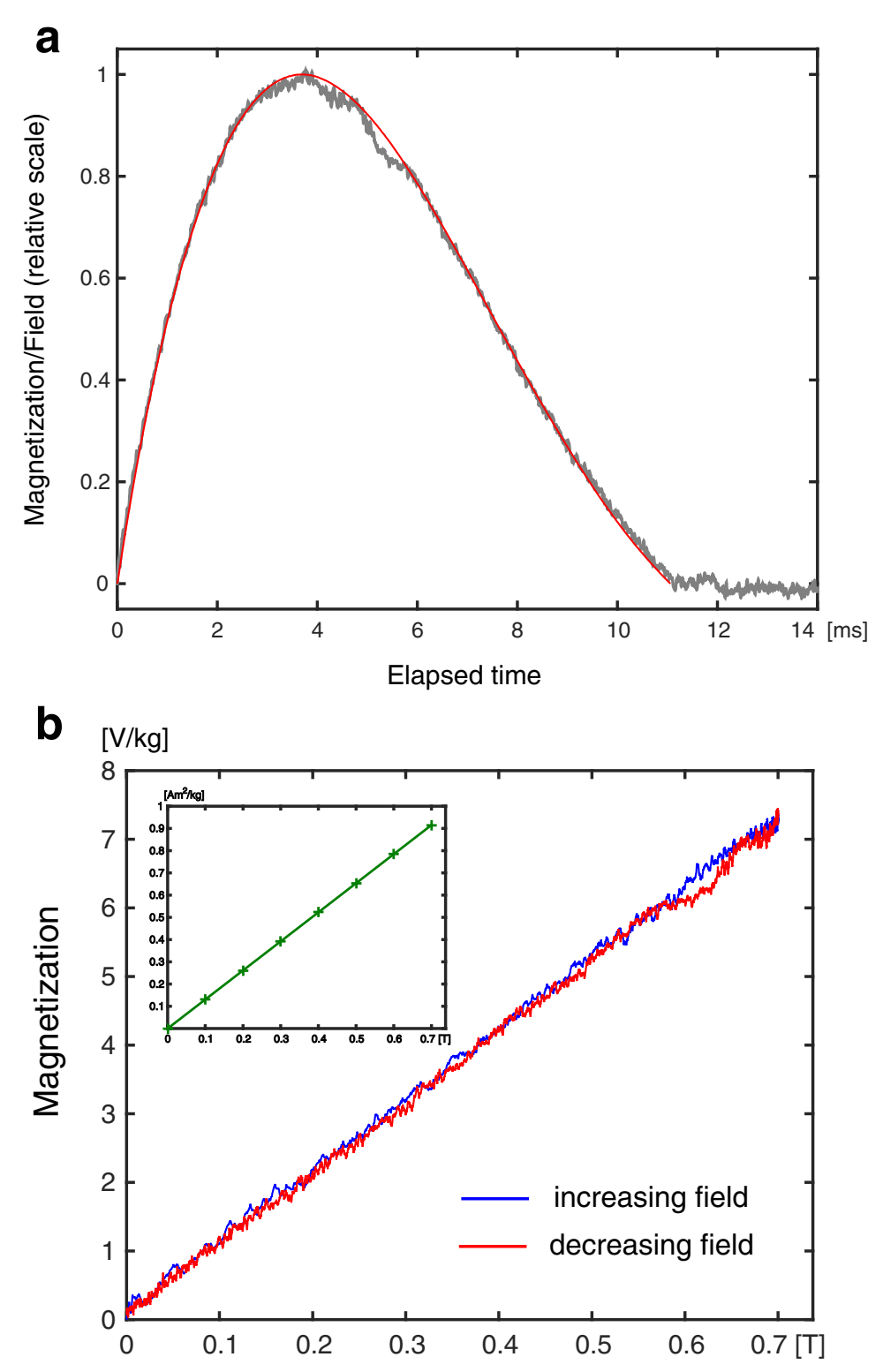

Field

Fig. 2 a $M-t$ curve of pulsed-field (red) for the duration of 11 ms and that of $\mathrm{Gd}_{2} \mathrm{O}_{3}$ (gray) for $14 \mathrm{~ms}$. b $\mathrm{M}-\mathrm{H}$ curve of $\mathrm{Gd}_{2} \mathrm{O}_{3}$ for field increasing (b/ue) and decreasing (red). The straight paramagnetic trend is consistent with results obtained by MPMS (inset)

systematic differences in the irreversibility are due to the fact that the curves in Fig. 5 are instantaneous records of the magnetization variations with time. This suggests that if using a longer pulsed field, the resulting $M-H$ curve would become more reversible and approach the hysteresis curve measured with a VSM. Conversely, if using a shorter pulse, the irreversibility will become more enhanced. In terms of the magnetic relaxation time $\tau$, the assumed time-dependence in the $M-H$ curve suggests that $\tau$ becomes longer as the measurement time-scale shorter.
Similar phenomena have been observed by the study of high-frequency hysteresis of SP-sized nanoparticles (Eggeman et al. 2007). They measured hysteresis loops of synthetic magnetite nanoparticles by changing the applied field frequency from $1 \mathrm{up}$ to $100 \mathrm{kHz}$. The resulting hysteresis loops are completely reversible as expected at lower frequencies, but, as the frequency increases to $30 \mathrm{kHz}$, they become irreversible and the opening of the loops is more visible. These frequency-dependent hysteresis loops could explain the slight opening of the $M-H$ curves in Fig. 5. However, to better explain the 


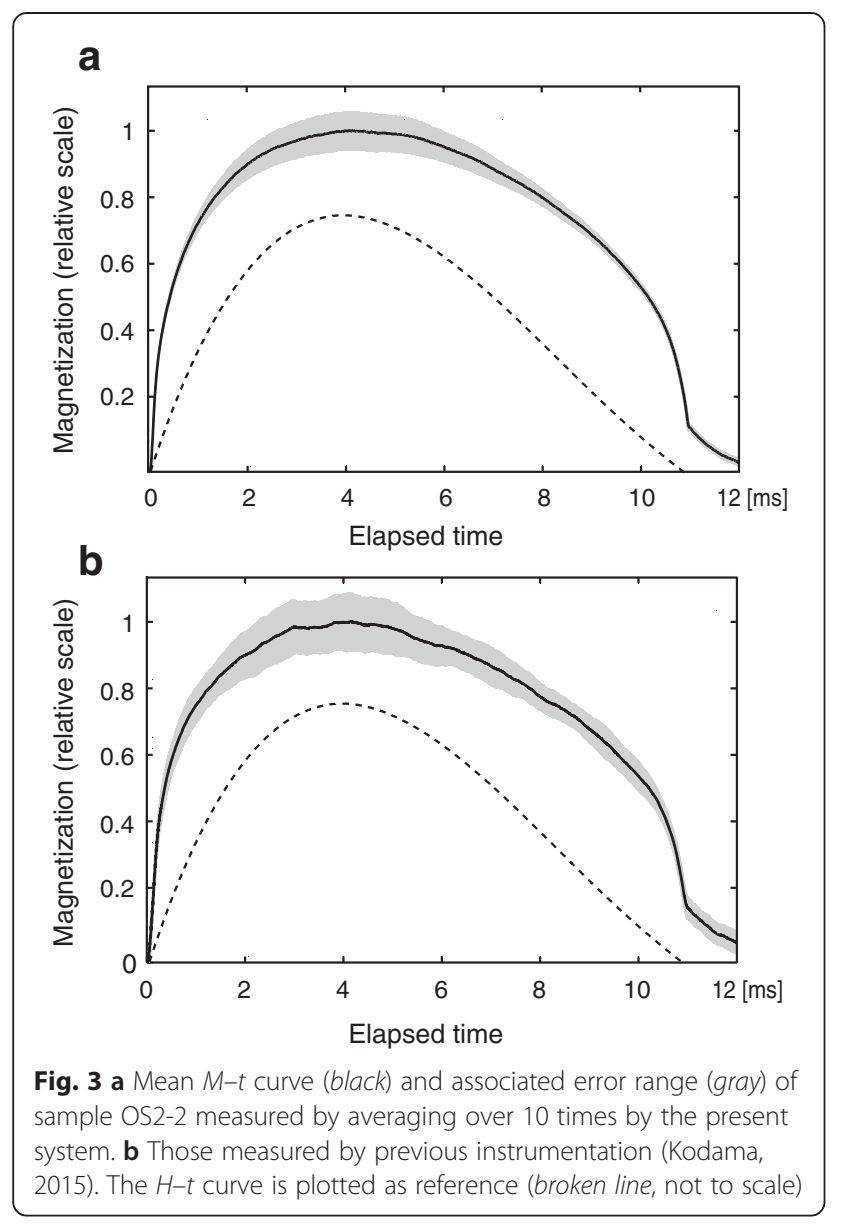

relationship between the irreversibility of the $M-H$ curves and frequencies, it is necessary to measure relaxation times in the time range of milliseconds by changing the field intensity. For this purpose, the $M-t$ curves in Fig. 4 were analyzed with the focus on their portions after turning off the field, and the results are described in the discussion section. In addition to the frequency-dependence of SD particles, MD particles could also be responsible for the enhanced irreversibility of the $M-H$ curves. This is most likely the case for sample SH03-07 in which MD particles are dominant. The opening of the $M-H$ curve below $0.4 \mathrm{~T}$ may indicate the energy dissipation by the rapid nucleation, pinning, and displacement of domain wall (DW) during the pulsed-field.

\section{Discussion}

The tail-off of the $M-t$ curves in Fig. 4 is plotted in Fig. 6, with a focus on the short interval after switching off a pulse. Each of them is superimposed by the highfrequency noise with increasing amplitudes with time. Despite the SNR increasing with time, the individual signal curves appear to fit well to an exponential curve with the form of $M_{0} \exp (-t / \tau)$, where $M_{0}$ is y-intercept, $t$ is time, and $\tau$ is the exponential decay constant, respectively. The estimated values of $\tau$ by least-squares curve fitting are listed in Table 1.

The previous estimation of $\tau$ for sample OS2-2 was $9.6 \pm 0.4\left(\times 10^{-4} \mathrm{~s}\right)$ (Kodama, 2015), $\sim 20 \%$ larger than the value in Table 1 . However, it is reasonable to conclude that the new values in Table 1 are more accurate

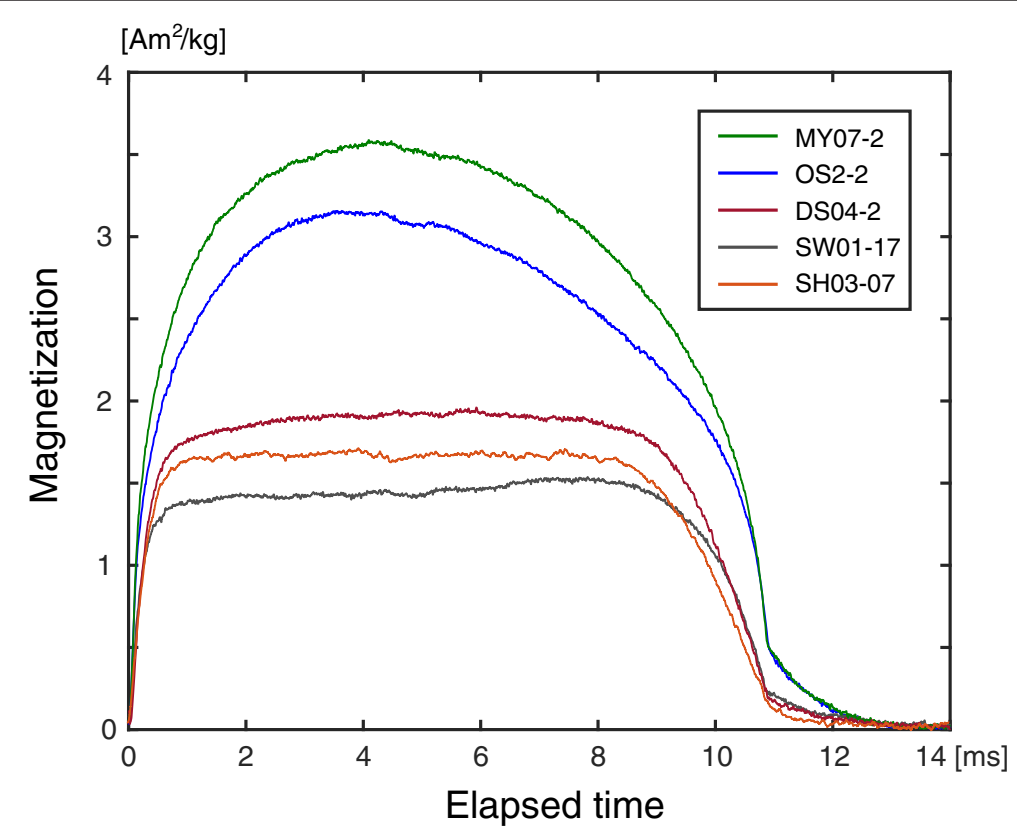

Fig. $4 \mathrm{M}$-t curves of the four volcanic rocks (OS2-2, SW01-17, MY07-2, DSO4-2) and one granite (SH03-07) 


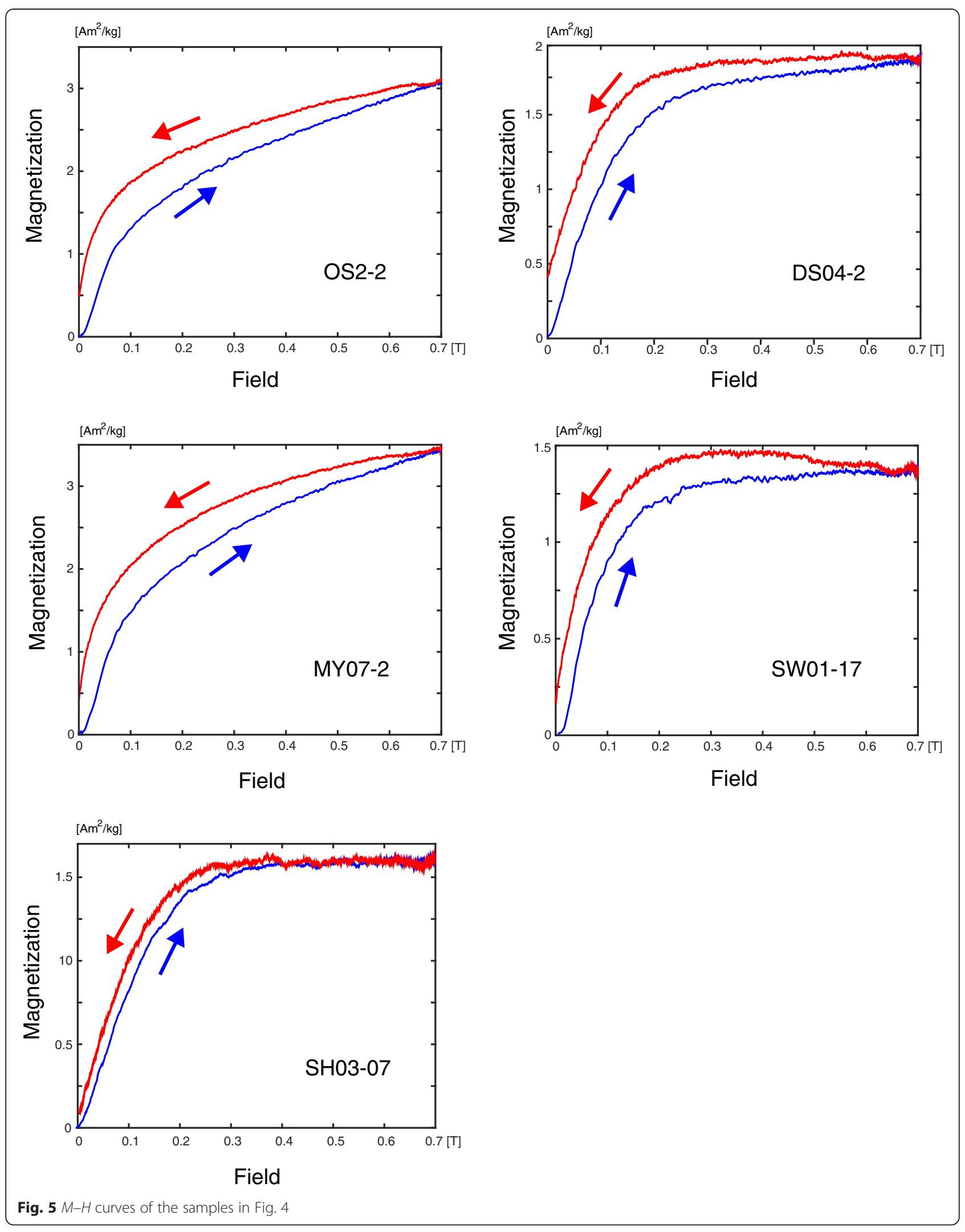




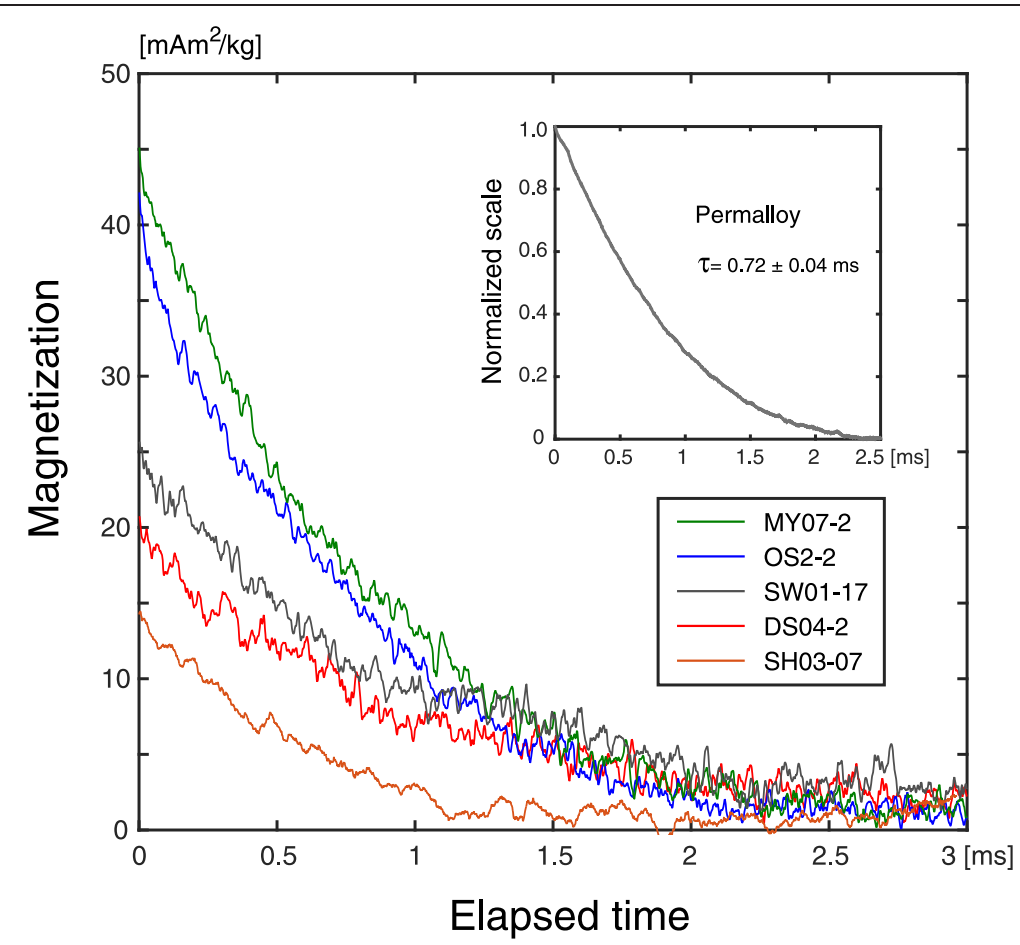

Fig. $6 M$ - $t$ curves of the samples in Fig. 4, focusing on the short interval (3 ms) after turning off a pulse. (Inset) $M-t$ curve of a permalloy sample (Kodama, 2015) measured with the present system. Plotted in normalized scale due to the large $M_{s}\left(40 \mathrm{Am}^{2} / \mathrm{kg}\right)$

than those obtained by the previous system because of the fact that the errors associated with $\tau$ are $<1 \%$.

It is noted in Table 1 that the samples are classified into two groups in terms of $\tau$. Referring to Figs. 4 and 5 , the one group with $\tau \sim 0.8 \mathrm{~ms}$ (MY07-2 and OS2-2) reached no saturation due to the coexisting paramagnetic contribution. This may lead to a conclusion that the more rapid decay for this group was caused to some extent by the paramagnetic particles present in these samples. However, theoretically (e.g., Dormann et al. 1996), the relaxation time for an ensemble of paramagnetic particles generally is $<10^{-9} \mathrm{~s}$ at room temperature, which is too small for the present system to detect. This indicates that the relaxation phenomena in Fig. 6 are free from the effect of paramagnetic particles but are

Table 1 Relaxation time $(\tau)$ of the fitted exponential decay curves in Fig. 6

\begin{tabular}{lc}
\hline Sample ID & $\tau\left(\times 10^{-4} \mathrm{~s}\right)$ \\
\hline MY07-2 & $7.89 \pm 0.03$ \\
OS2-2 & $7.60 \pm 0.03$ \\
SW01-17 & $11.66 \pm 0.08$ \\
DS04-2 & $11.45 \pm 0.09$ \\
SH03-07 & $7.72 \pm 0.15$ \\
Permalloy $^{\text {a }}$ & $7.20 \pm 0.04$ \\
\hline
\end{tabular}

aThe permalloy sample is the same as measured by Kodama (2015) and the $\tau$ was recalculated using the system in this study for comparison likely to be caused by the small fractions of superparamagnetic (SP) particles with the relaxation time in the narrow range of $10^{-4}$ to $10^{-3} \mathrm{~s}$. Alternatively, they could be due to an irreversible displacement of DWs, which generally yields characteristic relaxation time of $\sim 10^{-3} \mathrm{~s}$ for synthetic materials (Betancourt 2010). Because these volcanic rock samples contain more or less MD particles, the difference in $\tau$ in Table 1 may reflect magnetic property variations of the MD particles. It is also probable that the unsaturation of MY07-2 and OS2-2 might be due to magnetically hard fractions that are left unsaturated in a field $<0.7 \mathrm{~T}$, such as Ti-rich titanomagnetites present in mid-ocean ridge basalt (e.g., Fabian 2006). This being the case, such hard fractions may also contribute the slight opening of the $M-H$ curves in Fig. 5. To better understand the origin of these relaxation processes, it will be necessary to conduct combined analyses of magnetometry using both pulsed-field and static field, as well as various characterization methods in time domain (e.g., Weaver and Kuehlert 2012; Kolhatkar et al. 2013) and frequency domain (e.g., Astalan et al. 2007; Kodama 2013). Theoretically, the data in the time domain can be converted into the frequency domain by the Fourier transformation, or vice versa, by the inverse Fourier transformation. Thus, it is hoped that measurements in the two domains will be made for a sample to double-check the data in the respective domain. To achieve this, it is necessary to measure in the time domain of the microsecond range as well as in 
the frequency domain over a $100-\mathrm{kHz}$ range. Moreover, to apply the linear response theory, both measurements need to be done in weak fields. These requirements necessitate new instrumentation and methodology that are different in many aspects from those of the present study. The prototype system is being developed, and the results will be reported in a separate paper.

It should be pointed out that each $\tau$ in Table 1 is not a mean relaxation time of the entire magnetic particle assemblage. The width and range of the spectrum generally depend on the magnetic property and grain size, but the $\tau$ calculated in this study can be interpreted as the smallest relaxation time belonging to one broad spectrum of distribution. Thus, the separation of $\tau$ into two in Table 1 suggests that samples MY07-2 and OS2-2 have the minimum relaxation time longer than that of the other two. This imposes constraints on the assessment of the distribution of $\tau$ and, given proper assumptions of magnetic properties governing $\tau$, will lead to estimation of the grain size distribution. However, it is a serious drawback of the present system that the maximum field is limited to $0.7 \mathrm{~T}$ that is insufficient to saturate anti-ferromagnetic minerals such as hematite and goethite. To resolve this problem, it is necessary to lower the impedance of the pick-up coil by reducing the number of windings. But, the trade-off is the lower sensitivity that will require more improvement in the electronics. Another constraint is that it is hard for the present system to assess $\tau$ over the values in Table 1 , due to the limitations caused by the pulse duration and the resolution of measurement time that is $10^{-6} \mathrm{~s}$ in this study. To obtain a broader spectrum of relaxation time distribution, it is necessary to choose an appropriate method depending on the values of $\tau$ to be measured: the pulse magnetometry (this study) is suitable for estimation of $\tau<1 \mathrm{~s}$, and, above that, other methods using the time-dependence of IRM and VRM will be necessary (Worm 1999; Machac et al. 2007).

The benefit of the present system is that it uses a commercial pulse magnetizer, an instrument available in many paleomagnetism laboratories. Users have only to attach the sensing coil set into the sample cavity of the pulse magnet. The adoption of the analog circuit for preprocessing the coil output improved the sensitivity and made the following numerical processing flexible. We used in this study a digital oscilloscope for the data acquisition, but this can instead be accomplished with a fast data logger at a more reasonable cost.

It is hoped to have the pulse magnetometry applicable to sediments. This is indeed a more difficult task than simply increasing the maximum peak over $1 \mathrm{~T}$. It is necessary to increase the sensitivity more than 3 orders of magnitude, and this will be technically impossible as long as a single pulse is used. One possible way is numerical processing of the signals obtained by the application of the number of consecutive short pulses. To achieve this, state-of-the art techniques and methodologies for the characterization of synthetic magnetic nanoparticles for industrial and medical applications (e.g., Eggeman et al. 2007; Dennis and Ivkov 2013) would be helpful.

\section{Conclusions}

Instrumentation for measurements of dynamic magnetization induced by a pulsed-field was constructed. The fundamental framework of the new system is based on the previous system by Kodama (2015), and improvements were accomplished ensuring the sensitivity sufficiently high to measure a wide spectrum of igneous rocks. Results from a set of natural rock samples in the PSD to MD range indicate that the $M-H$ curves are comparable to the branch of the corresponding hysteresis loops measured in a static field. It appeared further that the irreversibility of the $M-H$ curves in the pulse were slightly more enhanced than in the static field. The opening of the $M-H$ curve might suggest the timedependence of the relaxation time of SP particles, the inclusion of high coercivity fractions of SD particles, and/or the energy dissipation caused by the displacement of the domain walls in the MD particles present in these samples. The $M-t$ curves showed rapid exponential decay after switching off a pulse. The estimated decay constants are in the order of $10^{-4}$ to $10^{-3} \mathrm{~s}$. This short aftereffect can be interpreted as the magnetic relaxation caused by the ensembles of magnetic particles.

\section{Competing interests}

The authors declare that they have no competing interests.

\section{Acknowledgements}

This work was supported by the Monbusho Kakenhi No. 24540455 and 15H03718. The author is grateful to Y. Yamamoto for providing the volcanic rock samples. This is contribution PMG15-03 from the Center for Advanced Marine Core Research, Kochi University.

Received: 30 March 2015 Accepted: 15 July 2015

Published online: 31 July 2015

\section{References}

Abrajevitch A, Kodama K (2011) Diagenetic sensitivity of paleoenvironmental proxies: a rock magnetic study of Australian continental margin sediments. Geochemistry, Geophys Geosystems 12:n/a-n/a. doi:10.1029/2010GC003481 Astalan AP, Jonasson C, Petersson K, Jakob Blomgren J, Ilver D, Krozer A (2007) Magnetic response of thermally blocked magnetic nanoparticles in a pulsed magnetic field. J Magn Magn Mater 311:166-170. doi:10.1016/ j.jmmm.2006.10.1182

Betancourt I (2010) Magnetization dynamics of amorphous ribbons and wires studied by inductance spectroscopy. Materials (Basel) 4:37-54. doi:10.3390/ma4010037

Dennis CL, Ivkov R (2013) Physics of heat generation using magnetic nanoparticles for hyperthermia. Int J Hyperth 29:715-729. doi:10.3109/02656736.2013.836758 Dormann JL, D'Orazio F, Lucari F, Tronc E, Prené P, Jolivet JP, Fiorani D, Cherkaoui $R$, Noguès $M$ (1996) Thermal variation of the relaxation time of the magnetic moment of $y$-Fe2O3 nanoparticles with interparticle interactions of various strengths. Phys Rev B 53:14291-14297

Dudding J, Knell P, Dudding J, Knell P a A, Cornelius RNNN, Enzberg-Mahlkeb B (2002) A pulsed field magnetometer for the quality control of permanent 
magnets. J Magn Magn Mater 242-245:1402-1404. doi:10.1016/S03048853(01)01247-1

Dudding J, Cornelius R, Knell P, Knell P, Lin A, and Jian H (2006) Improved repeatability of pulsed field magnetometry measurements through Peltier effect temperature control of measurement coils. J Iron Steel Res Int 13:388-392

Eggeman AS, Majetich SA, Farrell D, Pankhurst QA (2007) Size and concentration effects on high frequency hysteresis of iron oxide nanoparticles. J Appl Phys 43:2451-2453. doi:10.1109/TMAG.2007.894127

Egli R (2004) Characterization of individual rock magnetic components by analysis of remanence curves. 2. Fundamental properties of coercivity distributions. Phys Chem Earth 29:851-867. doi:10.1016/j.pce.2004.04.001

Fabian K (2006) Approach to saturation analysis of hysteresis measurements in rock magnetism and evidence for stress dominated magnetic anisotropy in young mid-ocean ridge basalt. Phys Earth Planet Inter 154:299-307. doi:10.1016/j.pepi.2005.06.016

Grössinger R (1982) Pulsed fields: generation, magnetometry and application. J Phys D Appl Phys 15:1545-1608

Grössinger R, Jewell GW, Dudding J, Howe D (1993) Pulsed field magnetometry. IEEE Trans Magn 29:2980-2982

Haase J, Eckert D, Siegel H, Müller, KH Eschrig, H Simon, A (2004) NMR in pulsed high magnetic fields. J Magn Magn Mater 272-276:2003-2005. doi:10.1016/ j.jmmm.2003.12.951

Heslop D, Dekkers MJ, Kruiver PP, Van Oorschot IHM (2002) Analysis of isothermal remanent magnetization acquisition curves using the expectation-maximization algorithm. Geophys J Int 148:58-64

Kapitza PL (1924) A method of producing strong magnetic fields. Proc R Soc A Math Phys Eng Sci 105:691-710. doi:10.1098/rspa.1924.0048

Kodama K (2013) Application of broadband alternating current magnetic susceptibility to the characterization of magnetic nanoparticles in natural materials. J Geophys Res. doi:10.29/2012JB009502

Kodama K (2015) Measurement of dynamic magnetization induced by a pulsed field: proposal for a new rock magnetism method. Front Earth Sci 3:1-9. doi:10.3389/feart.2015.00005

Kolhatkar AG, Jamison AC, Litvinov D, Wilson RC and Randall Lee T (2013) Tuning the magnetic properties of nanoparticles. Int J Mol Sci 14:15977-16009. doi:10.3390/ijms140815977

Kruiver PP, Dekkers MJ, Heslop D (2001) Quantification of magnetic coercivity components by the analysis of acquisition curves of isothermal remanent magnetisation. Earth Planet Sci Lett. 189:269-276

Likhite SD, Radhakrishnamurthy C, Murthy GT, Nagarajan R (1985) A simple method for the determination of the hysteresis parameters of hard magnetic materials by pulsed fields. J Phys D Instrum 18:401-404

Ludwig R, Bretchko P, Makarov S (2002) Magnetic and eddy current effects in an open-loop pulsed hysteresis graph system for magnetization of rare-earth magnets. IEEE Trans Magn 38:211-220

LV YL, Peng T, Wang GB, Ding TH, Han XT, Pan Y (2013) Magnet design and analysis of a 40 tesla long pulse system energized by a battery bank. J Low Temp Phys 170:475-480. doi:10.1007/s10909-012-0670-z

Machac TA, Zanner CW, Geiss CE (2007) Time dependent IRM acquisition as a tool to quantify the abundance of ultrafine superparamagnetic magnetite in loessic soils. Geophys J Int 169:483-489. doi:10.1111/j.1365-246X.2007.03355.x

Meier B, Greiser S, Haase J, Herrmannsdörfer T, Wolff-Fabris F, Wosnitza J (2011) NMR signal averaging in 62 T pulsed fields. J Magn Reson 210:1-6. doi:10.1016/j.jmr.2011.02.007

Miura N (2007) Physics of semiconductors in high magnetic fields. Series on semiconductor science and technology 15. Oxford University Press, Oxford.

Mizutani U, Oka T, Itoh Y, Yanagi Y, Yoshikawa M, Ikuta H (1998) Pulsed-field magnetization applied to high-Tc superconductors. Appl Supercond 6:235-246

Nojiri H, Choi K-Y, Kitamura N (2007) Manipulation of the quantum tunneling of nanomagnets by using time-dependent high magnetic fields. J Magn Magn Mater 310:1468-1472. doi:10.1016/j.jmmm.2006.10.616

Radhakrishnamurthy C, Likhite SD (1987) A pulsed field technique for the study of magnetic grains in rocks. Phys Earth Planet Inter 46:259-262

Takeyama S, Sakakura R, Matsuda YM, Miyata A, Tokunaga M (2012) Precise magnetization measurements by parallel self-compensated induction coils in a vertical single-turn coil up to 103 T. J Phys Soc Japan 81:1-7. doi:10.1143/ JPSJ.81.014702

Weaver JB, Kuehlert E (2012) Measurement of magnetic nanoparticle relaxation time. Med Phys 39:2765-2770. doi:10.1118/1.3701775

Worm H-U (1999) Time-dependent IRM: a new technique for magnetic granulometry. Geophys Res Lett 26:2557-2560

Yamamoto Y, Hoshi H (2008) Paleomagnetic and rock magnetic studies of the Sakurajima 1914 and 1946 andesitic lavas from Japan: a comparison of the LTD-DHT Shaw and Thellier paleointensity methods. Phys Earth Planet Inter 167:118-143. doi:10.1016/j.pepi.2008.03.006

Yamamoto Y, Shibuya H, Tanaka H, Hoshizumi H (2010) Geomagnetic paleointensity deduced for the last 300 kyr from Unzen Volcano, Japan, and the dipolar nature of the Iceland Basin excursion. Earth Planet Sci Lett 293:236-249. doi:10.1016/j.epsl.2010.02.024

\section{Submit your manuscript to a SpringerOpen ${ }^{\odot}$ journal and benefit from:}

- Convenient online submission

Rigorous peer review

- Immediate publication on acceptance

- Open access: articles freely available online

- High visibility within the field

- Retaining the copyright to your article

Submit your next manuscript at $>$ springeropen.com 